\title{
MODELING OF LICENSED PCS SELF-INTERFERENCE
}

\author{
T. Riley \\ National Telecommunications and Information Administration \\ Institute for Telecommunication Sciences \\ 325 Broadway \\ Boulder, CO 80305
}

\begin{abstract}
Self-interference in Personal Communications Services (PCS) systems is a real and acknowledged problem in both urban and rural areas. If left unresolved, it can impact the quality of service and system capacity. Self-interference is of particular interest to the commercial PCS industry, as more systems are being developed and implemented in already saturated areas. Since commercial PCS services are being considered for use in national security/emergency preparedness (NS/EP) situations, self-interference is becoming a concern of government and military agencies. A model of self-interference in PCS systems, which is applicable to other cellular, wireless technologies, has been developed by the Institute for Telecommunication Sciences (ITS). The developed methodology was applied to two existing PCS technologies: PCS 1900 (a narrowband time division multiple access (TDMA) system based on Global System for Mobile (GSM)), and IS-95-based code division multiple access; application to the remaining second-generation, as well as proposed second-and-a-halfand third-generation technologies is currently under way. The system-specific models are used to produce output noise and interference waveforms suitable for implementation in a real-time hardware channel simulator, or as a component of higher-level software simulations and models. Example outputs are given for simulations of both technologies, with corresponding statistical analyses of the noise and interference waveform properties. The models are particularly well-suited for independent PCS system evaluation by other Federal agencies, system manufacturers, and service providers.
\end{abstract}

\section{INTRODUCTION}

Personal Communications Services (PCS) has become the choice for mobile voice and data communication and is a significant resource during emergency recovery of telecommunication services following a natural disaster. PCS networks of varying technologies from many providers cover a majority of this nation's area. Most areas are serviced by multiple, non-interoperable systems which function independently but use the same radio frequency bands and infrastructure (base station sites and towers), contributing to one source of interference. When damages occur to the terrestrial telecommunication system, users tend to migrate to cellular resources, resulting in another source of interference. This sudden influx of traffic by private, commercial, civil, and Federal users results in wireless system overloads, interference, and disruption of service in the affected area. NS/EP planners and network operators must understand these interference effects to operate effectively in an overloaded environment. To aid in their understanding, the interfering environment caused by large numbers of active users and competing technologies must be characterized.
Currently, PCS service implementation uses a "first-out captures the market" philosophy. Due to market pressures, equipment manufacturers and service providers do not adequately characterize their own equipment, much less consider its effect on the equipment of other providers. A typical response by a service provider who experiences interference from adjacent or co-located services is to increase power. This is not effective in many cases and leads to an escalation of interference as other service providers increase power to offset the increased interference they experience.

Several standard propagation models are accepted by industry members (Okumura and COST-231/Walfish/ Ikegami) but no interference models have been developed or accepted. In 1997, ITS implemented a PCS network evaluation program that provided performance metrics, interoperability studies, and scenario plans for present and future PCS system operation [1]. The initial development covered system-specific interference models, implementable in hardware, for two licensed PCS technologies: PCS 1900 (a narrowband time division multiple access (TDMA) system based on Global System for Mobile (GSM)), and code division multiple access based on IS-95. The model covers the interference generated by adjacent or co-located PCS systems on one another. It takes into account system considerations and management functions (such as power control in IS-95) that are affected by the dynamic nature of the interference.

The generic model involves a hexagonally arranged circular cellular geometry, with each cell having six adjacent cells (Figure 1). A single base station is located in the center of each cell and any number of mobile stations are randomly located within a cell's radius. The interference model consists of four components:

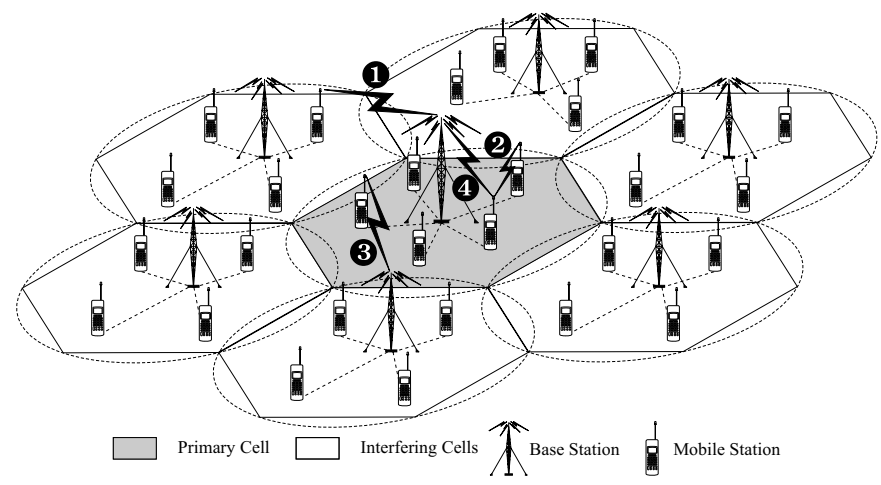

Figure 1. Generalized model geometry and self-interference components. 
Base-to-mobile link:

(1) victim base station is interfered with by mobile stations in adjacent cells.

(2) victim base station is interfered with by mobile stations in the same cell.

Mobile-to-base link:

(3) victim mobile stations are interfered with by base stations in adjacent cells.

(4) victim mobile stations are interfered with by the base station in the same cell (for IS-95 systems only).

The output of the model consists of voltage envelope, power envelope, and phase distribution histograms of the aggregate interference waveform. This information can be used as input to a hardware simulator or a software modeling program to evaluate system designs or actual hardware implementations. It also can be used to characterize one-on-one, one-on-many, and many-on-one interference. As a result, potential solutions can be proposed; these solutions can be used by field personnel to solve existing problems or to anticipate and avoid potential problems.

Since the development of the model began, the communications industry has developed and proposed new technologies to address system limitations such as system capacity and coverage, and data transfer rates. In the near-term, 2.5G systems such as EDGE and GPRS are enhancements to current technologies designed to improve current services without requiring extensive changes to the existing infrastructure. In the long-term, $3 \mathrm{G}$ systems such as cdma2000 and W-CDMA (UTRA in Europe) have been proposed to support the goals established by the International Telecommunication Union (ITU) with IMT-2000. In addition, high-altitude platform systems and unmanned air vehicles (UAVs) will have an effect on existing systems due to their large signal footprint and effective radiated power. As a result of these advances, ITS is currently expanding the model to accommodate these upcoming systems and to determine the effects that co-located or adjacent PCS systems have on one another.

\section{CONSIDERATIONS}

Complete specification of the PCS RF channel must include a credible model of the system-specific noise and interference $(N / I)$ environment. Incidental channel impediments are termed "noise," and originate from both natural and artificial sources. "Interferers," by contrast, are waveforms intentionally radiated in the RF channel that disrupt or degrade a desired waveform. Interferers are generated by sources both external and internal to the affected PCS system, and may include other wireless systems sharing a common geographical area and frequency band.

Preliminary studies of the noise environment at $2 \mathrm{GHz}$ show that the natural noise contribution is relatively small [2]. Interferers, by contrast, can be a major hindrance to wireless communication at $2 \mathrm{GHz}$. This is particularly true in a multiple access environment using microcellular deployment. PCS system capacity is thus interferencelimited.

PCS systems are largely untested in actual deployment scenarios. Because predeployment field testing of all aspects of an actual PCS system is prohibitively time-consuming and expensive, other evaluation methods are required. These include real-time hardware simulation of the PCS channel and software simulation of the PCS network. Hardware simulation of the PCS channel provides an economical, controlled environment for testing PCS equipment at the link-level. In addition, link- and network-level software simulation techniques can be used to provide predictions of PCS system performance.

For hardware implementation, a quantitative description of the interference waveform is required. Unfortunately, most descriptions of the interference environment in the literature present statistical models of the interference waveform behavior, rather than a model of the waveform itself. However, complex multiple access waveforms from numerous mobile stations may create an interference environment that cannot be described by a single distribution function.

Development of a real-time testing capability involves software simulation of the interference waveform, then adaptation of the results to hardware capable of real-time waveform processing. Ideally, model development and validation should be conducted by an independent PCS system evaluator. Models should be cross-compatible for each of the PCS technologies under test.

\section{PCS CELLULAR GEOMETRY AND GENERIC INTERFERENCE EXPRESSIONS}

Development of the ITS intrasystem interference waveform based on spatial considerations includes specification of a generic PCS cellular system geometry, i.e., one suited to either IS-95 or TDMA. A hexagonally arranged circular cellular geometry is used, with each cell having six adjacent cells. The following assumptions apply to the PCS cellular geometry:

1. All cells within the PCS system are the same size.

2. A single base station is located at the center of each cell.

3. Each cell contains $N_{U}$ mobile stations.

4. One RF carrier is simulated per cell.

5. A "primary cell" contains the base station or mobile stations encountering interference.

The primary cell is the focus of the interference waveform simulation. On the base-to-mobile link, it contains the base station which is experiencing interference from mobile stations outside the primary cell transmitting on the same RF frequency. On the mobile-to-base link, it includes nearby base station transmissions that interfere with mobile stations in the primary cell. 
$\overline{x_{F I(k n)}}(t)$ is the complex baseband expression for the interference waveform from the $n^{\text {th }}$ interferer in interfering cell $k$. Depending on the link direction, the interferer may be either a mobile station or a base station. For the base-to-mobile link, $n$ represents the $n^{\text {th }}$ interfering mobile station in the $k^{\text {th }}$ cell. For TDMA systems, $\overline{x_{F I}(k, n)}(t)$ is defined over one time slot on the base-to-mobile link. For other technologies, such as IS-95, $\overline{x_{F I(k, n)}}(t)$ may not be time-limited. For the mobile-to-base link, $n$ indicates the $n^{\text {th }}$ mobile station in the primary cell experiencing interference from the base station in interfering cell $k$. In the mobile-to-base link case, $\overline{x_{F L(k, n)}}(t)$ is defined over one frame for TDMA systems, and is not time-limited for IS-95 systems.

$\overline{x_{F I(k, n)}}(t)$ is the basic building block for the interference waveform expressions. In the $N / I$ model, it is the only expression referenced to the transmitter (i.e., all other expressions represent the interference waveform incident at the receiver). All other interference functions are expressed as summations of $\overline{x_{F I(k, n)}}(t)$. For example,

$\overline{x_{F I(k,-)}}(t) \quad$ is defined as the sum of all base-to-mobile link interferer mobile station waveforms created in interfering cell $k$, incident at the primary cell's base station.

$\overline{x_{F I}}(t) \quad$ is the aggregate base-to-mobile link interference waveform incident at the primary cell's base station.

$\overline{x_{F I(-, n)}}(t) \quad$ is the mobile-to-base link interference waveform caused by all interfering base stations, as seen by the $n^{\text {th }}$ mobile station in the primary cell.

Expressions for the base-to-mobile and mobile-to-base link interference functions depend on the technology. However, generic expressions can be formed with parameter specification of an IS-95- or TDMA-based technology. These generic expressions are shown below, with the parameter $b$ specifying IS-95 or TDMA.

For the base-to-mobile link,

$$
\begin{gathered}
\overline{x_{F I(k,-)}}(t)=\sum_{n=0}^{N_{U}-1} \overline{x_{F I(k, n)}}\left(t-n b T_{U}-\frac{r_{k, n}}{c}\right) a_{k, n}\left(r_{k, n}, \theta_{k, n}\right) \\
\overline{x_{F I}}(t)=\sum_{k=0}^{N_{I}-1} \overline{x_{F I(k,-)}}(t),
\end{gathered}
$$

and for the mobile-to-base link,

$\overline{x_{F I(-, n)}}(t)=\sum_{k=0}^{N_{I}-1} \overline{x_{F I(k, n)}}\left(t-\frac{r_{k, n}}{c}\right) a_{k, n}\left(r_{k, n}, \theta_{k, n}\right)$

where, for both Equations (1) and (2): $c$ is the speed of waveform propagation, $T_{U}$ is the duration of a TDMA time slot, $N_{U}$ is the number of mobile stations per cell, $N_{I}$ is the number of interfering cells, $r_{k, n}$ is the distance between the point interferer and the receiver, $\theta_{k, n}$ is the angle of the point interferer with respect to the receiver, $a_{k, n}\left(r_{k, n}, \theta_{k, n}\right)$ is a propagation loss and Doppler function, $r_{k, n} / c$ is the propagation delay, and $b$ indicates TDMA $(b=1)$ or IS-95 $(b=0)$.

Figures 2 and 3 show the base-to-mobile and mobile-to-base link relationships, respectively, between a single interferer and receiver. This geometry is used repeatedly to locate all interferers.

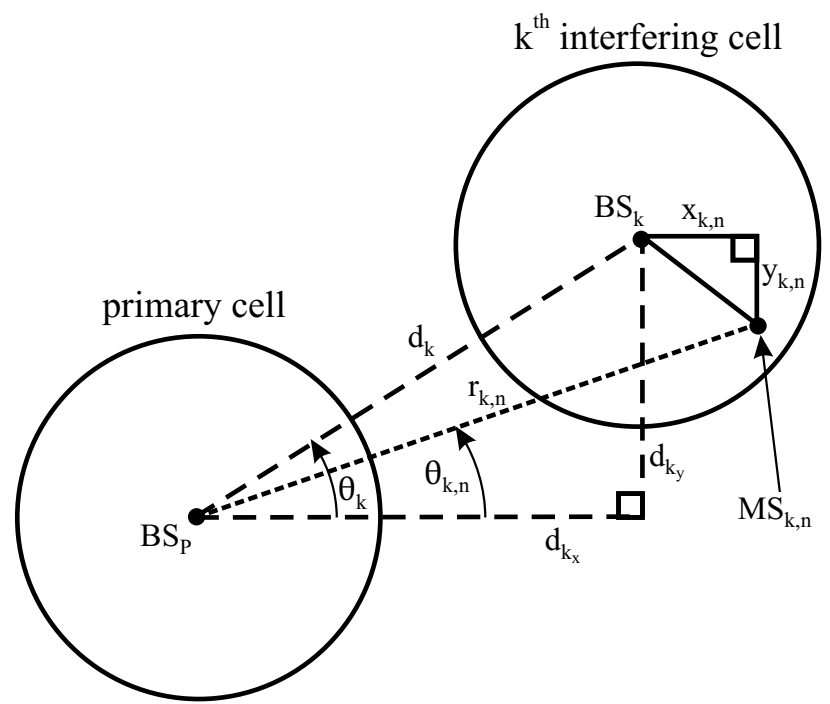

Figure 2. Base-to-mobile link interferer geometry.

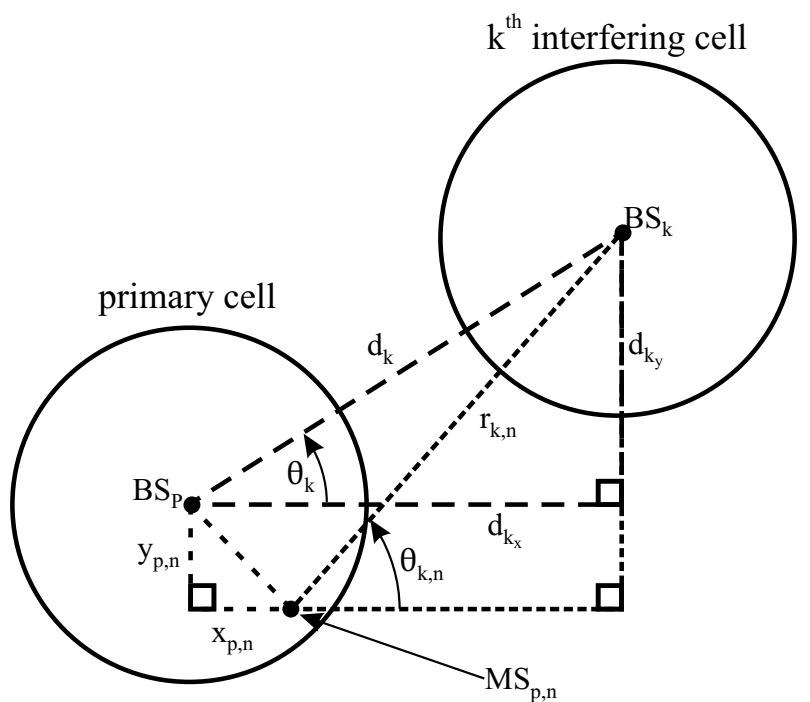

Figure 3. Mobile-to-base link interferer geometry.

\section{TYPICAL INTERFERENCE SIMULATION}

Simulation of base-to-mobile link interference results in the aggregate interference waveform caused by nearby interfering cells, as seen at the primary cell's base station receiver. A few assumptions were made to simplify the computations, and to randomize certain parameters as follows:

- All interfering cells for a given iteration of the program are equidistant from the primary cell's base station. 
- Each simulation iteration calculates the aggregate waveform from an equidistant layer of interfering cells. For example, the interference waveform from cells adjacent to the primary cell will be computed in one iteration, since all cells are an equal distance from the primary cell. Since two different distances are needed for the second layer of interfering cells, two iterations are required. The total waveform is computed by adding. several simulation iterations together. Obtaining the interference waveform due to interferers in both adjacent and second layer cells therefore requires three simulation iterations.

- All interfering cells transmit on the same RF carrier frequency. Cells not contributing to the overall interference waveform (e.g., resulting from a cell reuse pattern), may be "zeroed out" during the computation.

- $\quad$ Frames from interfering cells are aligned (this may be changed as a simulation parameter).

- Symbols are not aligned from interferer to interferer. A random $U(0, T]$ delay is incorporated in each time slot.

Many different $N$ / I scenarios may be created by setting the simulation parameters accordingly, and consequently a large number of different simulation studies may be conducted. Two simple cases drawn from the PCS 1900 TDMA model have been chosen to demonstrate the capabilities of the simulation tool. In case 1, six interfering cells, all adjacent to the primary cell, are simulated. Case 2 simulates fifty interfering cells that are all equidistant from the primary cell.

For case 2, significant overlap in coverage area occurs, while case 1 reflects a more realistic scenario. Three plots are created for each case, showing statistics of the aggregate interference waveform in the following order: 1) the voltage envelope histogram, 2) the power envelope histogram, and 3) the phase distribution histogram.

Results for Case 1. Figures 4, 5, and 6 show the voltage envelope, power envelope, and phase histograms, respectively, of the interference waveform caused by six adjacent interfering cells. The voltage envelope distribution in Figure 4 appears Rayleigh-distributed, although distorted in form. The large value at zero is caused by the TDMA guard times, and the simulation assumes that frames in adjacent cells are aligned (when frames are aligned, so are the guard times, resulting in a large number of zero values). Randomization of frame alignment in the simulation simply involves circularly rotating each interfering cell waveform component before aggregation.

Similarly, the power envelope distribution appears as a distorted exponential in Figure 5. The phase distribution of Figure 6 is roughly uniformly distributed on the interval $(-\pi, \pi]$. Again, the large count value at zero is caused by the guard times.

Results for Case 2. In Figures 7, 8, and 9, the number of simulated interfering cells was increased to fifty, with all other parameters the same as for case 1 . Note that the voltage envelope profile given in Figure 7 appears more

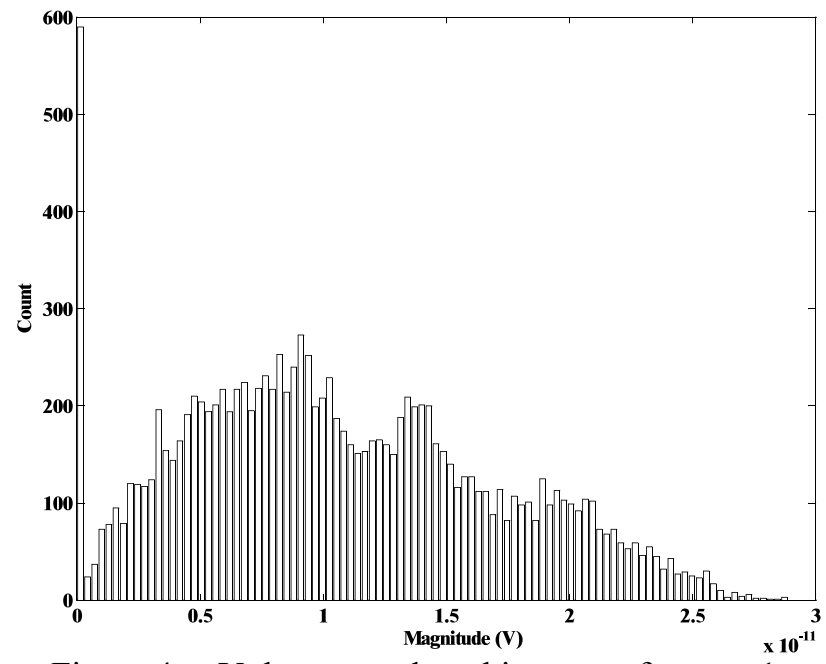

Figure 4. Voltage envelope histogram for case 1.

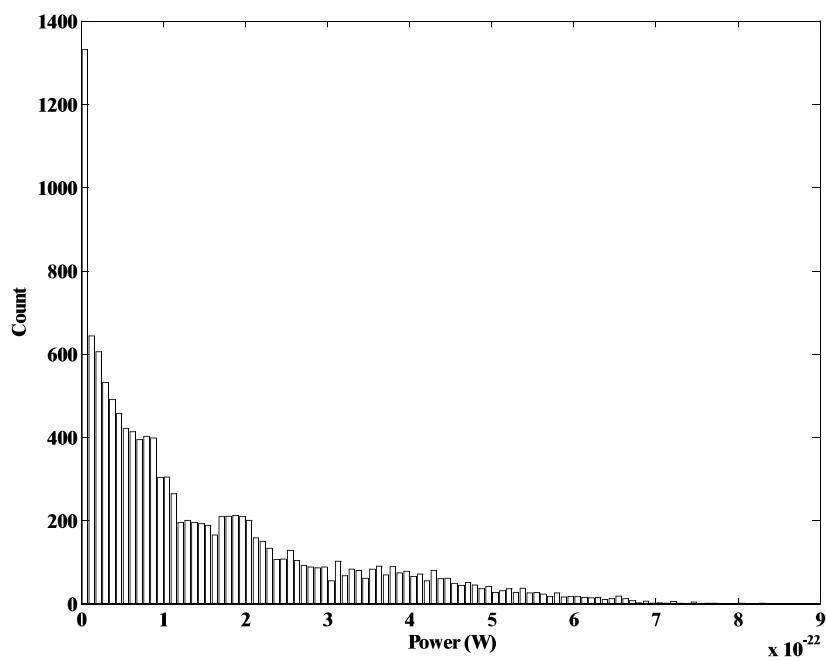

Figure 5. Power envelope histogram for case 1.

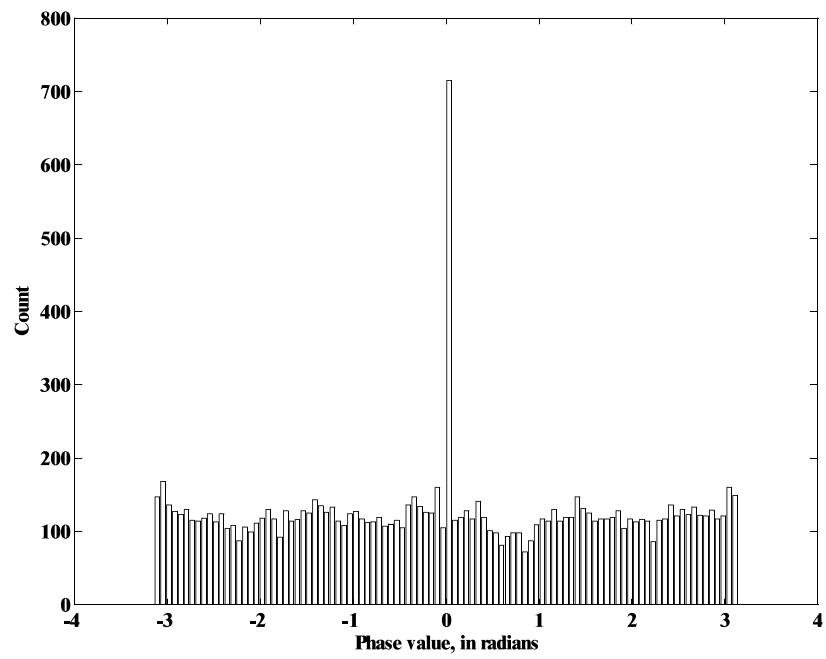

Figure 6. Phase envelope histogram for case 1. 


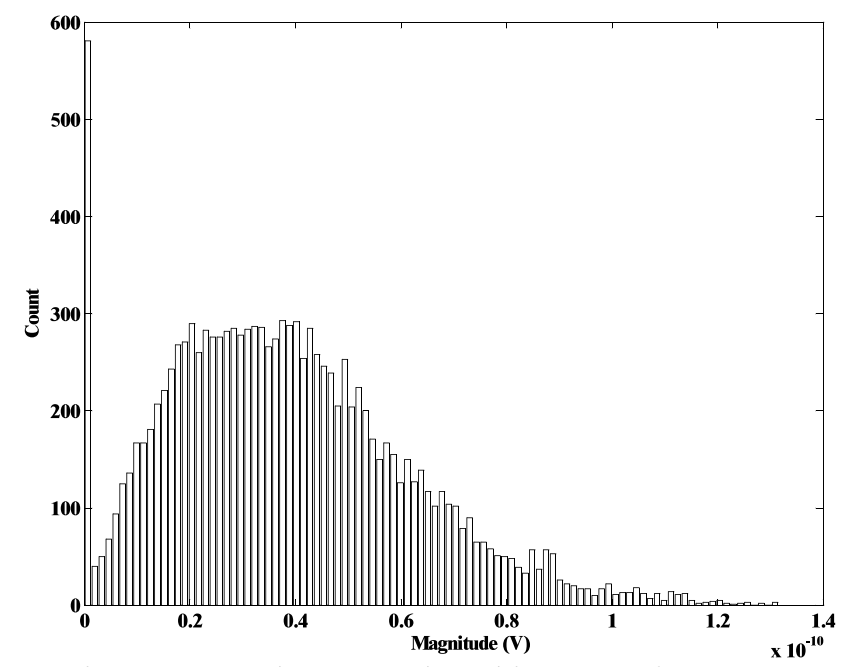

Figure 7. Voltage envelope histogram for case 2.

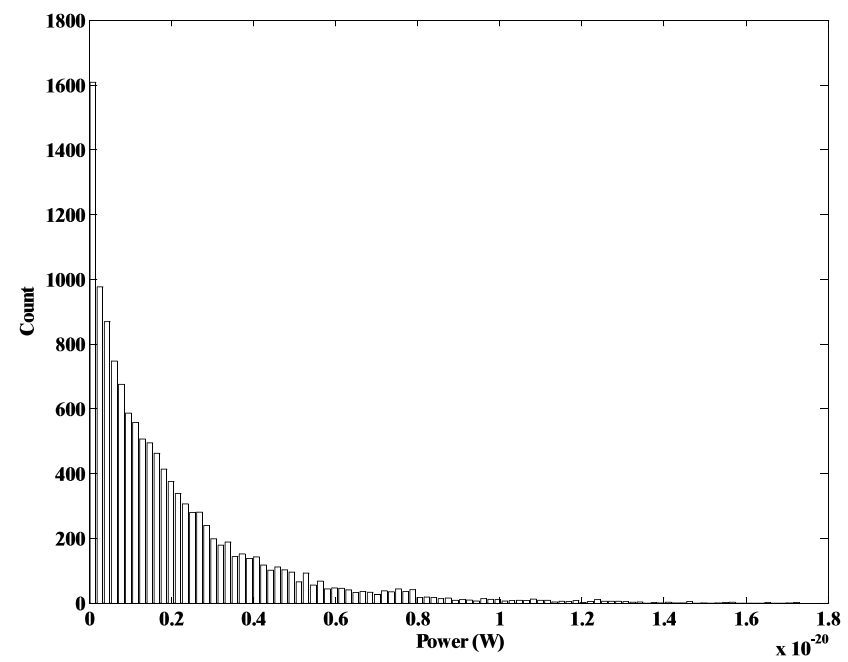

Figure 8. Power envelope histogram for case 2 .

Rayleigh-distributed, the power envelope in Figure 8 more exponential, and the phase distribution in Figure 9 more uniform than those calculated for case 1 .

Non-Gaussian Effects. By setting the power levels explicitly for each interfering mobile station, it is readily demonstrated that the interference waveform is not purely Gaussian. This is demonstrated for an extreme case in Figure 10, which shows the resulting distorted amplitude envelope when a single interfering mobile station in case 1 has its transmitted waveform multiplied by a factor of ten.

Outputs from the simulation may be used directly in a real-time hardware channel simulator by repeating an interference waveform of integer frame length. The number of samples per frame is flexible, and implementable in commerically-available waveform generators. The simulated waveform may also be abstracted using statistical methods, at the cost of losing some attributes inherent in the actual waveform.

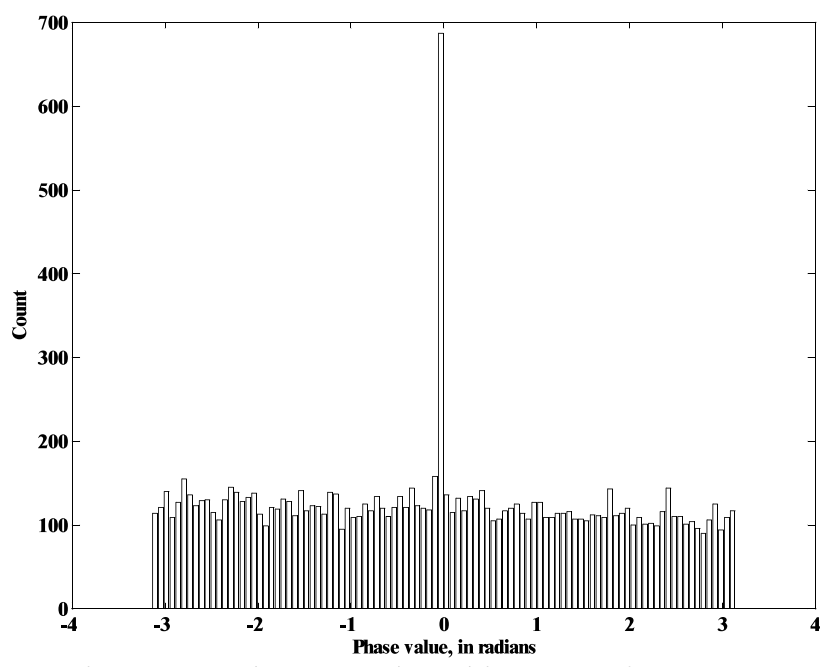

Figure 9. Phase envelope histogram for case 2.

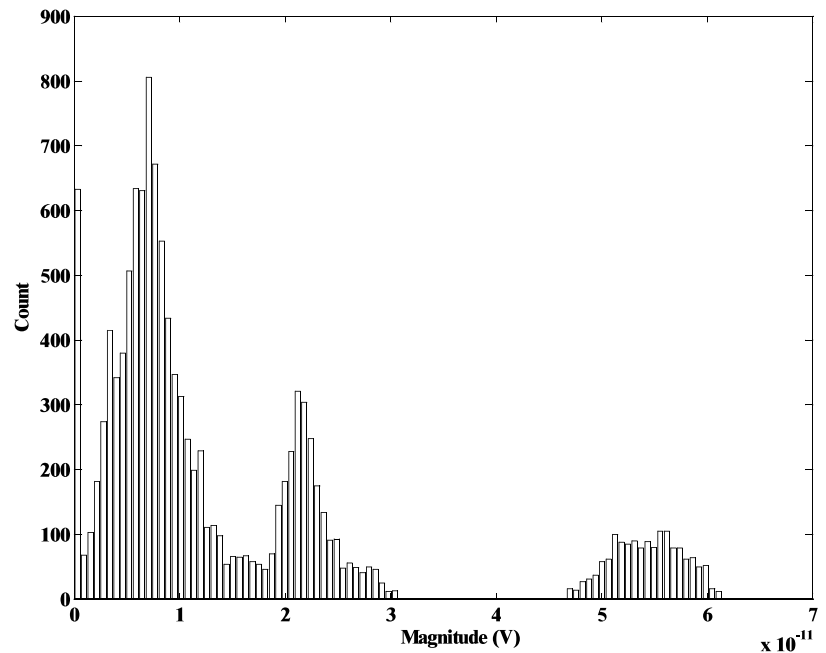

Figure 10. Voltage envelope for case 1, with the addition of a single dominant interferer.

\section{CONCLUSION}

$N$ / I models are essential for realistic characterization of the operating environment for proposed PCS technologies. As a justification, the examples demonstrate that many environmental and systematic influences affect the statistics of the aggregate interference waveform, and that a Gaussian distribution assumption is too simplistic in many cases. Channel simulation using these $N / I$ models provides a valuable PCS evaluation capability, and provides an alternative to expensive PCS field testing.

\section{REFERENCES}

[1] J. G. Ferranto, "Interference simulation for personal communications services testing, evaluation, and modeling," NTIA Report 97-338, July 1997.

[2] A.D. Spaulding, "The natural and man-made noise environment in personal communications services bands," NTIA Report 96-330 (revised), May 1996. 